\title{
The impact of COVID-19 on surgical training at a tertiary hospital in Greece: a 'hidden infectious enemy' for junior surgeons?
}

\author{
Michail Vailas (D) Maria Sotiropoulou · Francesk Mulita · Nikolaos Drakos · Elina Ambalov · loannis Maroulis
}

Received: 21 February 2021 / Accepted: 24 February 2021 / Published online: 8 April 2021

(c) Springer-Verlag GmbH Austria, part of Springer Nature 2021

\begin{abstract}
Summary
Background Soon after its appearance, the coronavirus disease 2019 (COVID-19) caused by severe acute respiratory syndrome coronavirus 2 (SARSCOV-2) became a pandemic, with over 111 million cases reported and 2.4 million deaths worldwide. Although the focus of public health systems must lie on patients' care and treatment, SARS-COV-2 infection has also affected surgical trainees in their academic and professional development, causing significant disruption in all forms of their training.

Methods The aims of this study were firstly to assess the impact of SARS-CoV-2 on core surgical training, regarding operative, educational and academic skills, as well as the general effect on psychological status and well-being of surgical trainees at a tertiary center in Greece. All core surgical trainees (17) in the general surgery department at the University Hospital of Patras were invited to participate in a voluntary anonymous survey via printed questionnaires.

Results Junior trainees and senior surgical trainees performed or assisted in almost $50 \%$ fewer cases in the COVID era when compared with the period reported before the virus. As far as courses, conferences and exams attended by trainees are concerned, a sig-
\end{abstract}

Michail Vailas and Maria Sotiropoulou contributed equally to this manuscript.

M. Vailas, MD $(\bowtie) \cdot$ F. Mulita, MD · N. Drakos, MD ·

E. Ambalov, MD · I. Maroulis, MD, PhD

Department of Surgery, University of Patras, University

Hospital of Patras, 26504 Patra, Greece

mike_vailas@yahoo.com

M. Sotiropoulou, MD

First Department of Surgery, National and Kapodistrian

University of Athens, Laikon General Hospital, 17 Agiou

Thoma, 11527 Athens, Greece

mike_vailas@yahoo.com nificant reduction in numbers (six vs 35) before and after the start of COVID-19 pandemic was apparent. In all, $10(62.5 \%)$ trainees felt that their confidence in the operating theatre had been negatively impacted by the pandemic, while four (25\%) trainees felt significant stress levels as a result of the national lockdown. Conclusions It is imperative for surgical educators to design and implement new alternative ways to assist surgical trainees in their education and also avoid their undertraining during the pandemic.

Keywords Coronavirus - Education - Pandemic . SARS-COV-2 $\cdot$ Surgery

\section{Introduction}

In late December 2019, the coronavirus disease 2019 (COVID-19), caused by severe acute respiratory syndrome coronavirus 2 (SARS-CoV-2), emerged in Wuhan, China, and soon after its appearance became a pandemic with over 111 million cases reported and 2.4 million deaths [1]. The World Health Organization declared the disease a pandemic on 11 March 2020, having affected over 100 countries worldwide [2]. Greece has already paid its toll with over 6000 deaths and thousands of hospital admissions, which test the capability of the national health care system every day.

Although the focus of public health systems must lie on patients' care and treatment, SARS-COV-2 infection has also affected surgical trainees in their academic and professional development, causing significant disruption in all forms of medical training and teaching [3]. Moreover, it seems that the pandemic also has a major impact on the stress and anxiety levels of health care professionals as well as young surgeons [4]. Some of the workforce, including consultants and trainees, has been redeployed to acute-care 
COVID-19 units and a significant proportion is in selfisolation due to suspicious symptoms or contact with patients positive for COVID-19.

In Italy, COVID-19 has been shown to have a detrimental effect on the training of urology registrars. The proportion of residents experiencing a severe reduction $(>40 \%)$ or complete suppression $(>80 \%)$ of training exposure ranged between $41.1 \%$ and $81.2 \%$ for "clinical" activities, and between $44.2 \%$ and $62.1 \%$ for "surgical" activities in a study of 351 patients [5]. In the UK as well as in Greece, normal training rotations have been suspended. There are few elective surgical theatre lists or clinics. Many educational events, including conferences and examinations, have been cancelled [6]. Problems and challenges that arise through COVID-19 for surgical training and education include cancellation of elective operations and disruption of elective operating room attendance, as well as cancellation of advanced surgical training courses and seminars. A lack interpersonal communications, social distancing, lockdown and associated mental health problems might contribute as major burdens in the education of young doctors [7].

Inevitably, the COVID-19 pandemic has been a major obstacle for the training process, with trainees unable to achieve the competencies required for their professional career. The current fear of generating a group of medical professionals under the term the "COVID Generation"-professionals without adequate and satisfactory training capabilities-is a terrible scenario, which in turn will affect patients' health even after the pandemic disappears. Taking into account the reduction in practical training skills and also the necessity to maintain social distancing, many have suggested alternative methods to support the training of young doctors such as web-based learning and participation in surgical simulation sessions. Despite the fact that studies have highlighted that there is no substitute for practical and hands-on experience, such alternative means might be the ideal tool for continuous learning for the time being [8, 9].

The aims of this study were firstly to assess the impact of SARS-CoV-2 on core surgical training, regarding operative, educational and academic skills, as well as the general effect on the psychological status and wellbeing of surgical trainees at a tertiary center in Greece.

\section{Methods}

\section{Study design}

The study was approved by the institutional research ethics committee before the investigation was started and has been conducted in accordance with the principles set out in the Declaration of Helsinki. Trainees in the general surgery department at the University Hospital of Patras were invited to anonymously and voluntarily complete a survey designed by the authors and collected on the 30th of November 2020. The survey consisted of 40 paper-printed questions regarding trainee demographics and years of training, operative cases performed or assisted, general surgical activity in the surgical department and emergency room department, confidence, academic activities, courses and conferences, exams and finally sickness and sense of wellbeing. Written permission was granted by the chief of the surgical department to conduct the study. Trainees were given the opportunity to decline or answer the number of questions they wanted. The purpose of the study was explained in detail and, through an invitation email, permission given for the anonymous results to be used in the current study.

\section{Statistical analysis}

Association between categorical variables was assessed using Chi-squared test. We considered a $P<0.05$ as statistically significant. All statistical analysis was performed using Statistical Package for Social Sciences (SPSS) version 16 for Windows (SPSS Inc, Chicago, IL).

\section{Results}

\section{Study population}

Of the 17 general surgery trainees sent the online survey, $16(94.1 \%)$ responded. A 6-year training period is obligatory in order to complete general surgery residency programs in the Greek National Health Care System. Three of 16 were in their first year of training and four of 16 in their fourth year. A total of $95 \%$ were in the age group between $24-30$ years and 5\% were 31-35 years old. Two (12.5\%) of the trainees were married and lived with a spouse. Fig. 1 depicts the number of trainees based on their years of surgical training.

\section{Number of operations performed or assisted}

In Fig. 2, the number of operations performed based on years of training $(1-2,2-4,4-6)$ in 4 -month periods before and after the start of the COVID-19 pandemic

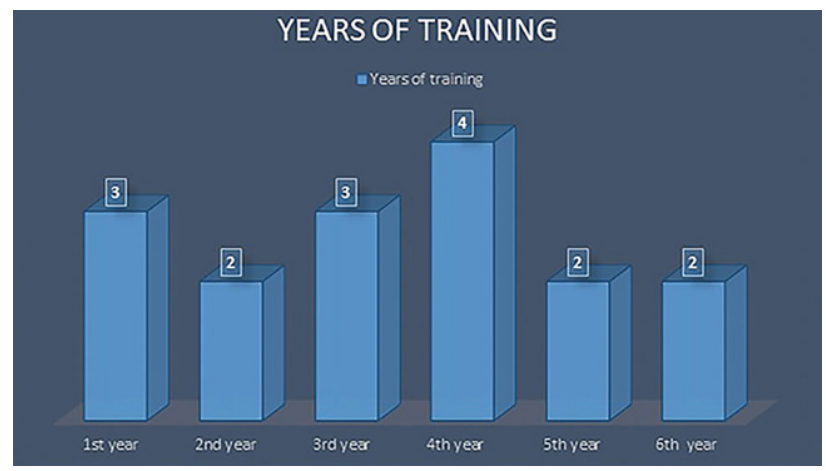

Fig. 1 Surgical trainees based on their years of training 
Fig. 2 Number of operations performed based on years of training in 4-month periods before and after the start of COVID-19

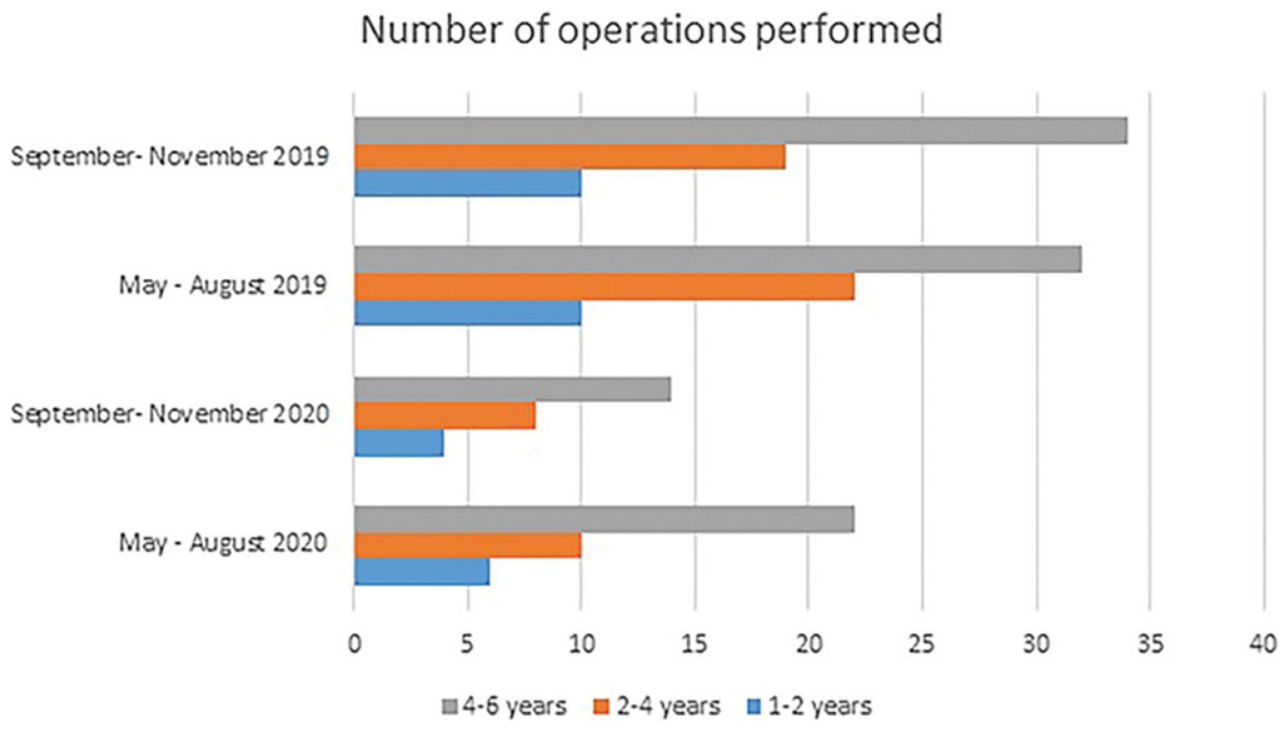

are presented. Junior trainees (1-2 years) and senior trainees (4-6 years) performed almost $50 \%$ fewer cases in the COVID era, when compared with the period reported before the virus. The same figure (a 50\% reduction) appears for junior doctors in operations as assisting surgeons, while senior doctors assisted in 37 cases during the pandemic and in 90 before the pandemic (Fig. 3).

\section{Courses, conferences and exams}

As far as courses, conferences and exams are concerned, Fig. 4 shows a significant reduction (six vs 35) before and after the start of COVID-19 pandemic. Since the pandemic, eight $(50.0 \%)$ trainees had not attended any teaching, while five (31.2\%) had their teaching re-delivered by webinars. Three (18.7\%) trainees had their pre-booked courses, conferences or exams cancelled due to the national lockdown.

\section{Academic activity: scientific writing}

Despite the fact that $13(81.2 \%)$ trainees did not report progress in their surgical portfolio in the SARSCoV-2 era, 10 (62.5\%) managed to write and publish more scientific papers compared with the time period before the virus. This is probably the result of reduced workloads during the pandemic.

\section{Confidence levels}

In all, $10(62.5 \%)$ trainees felt that their confidence in the operating theatre had been negatively impacted by the pandemic, while five $(31.2 \%)$ felt no difference (Fig. 5). As far as managing acutely ill and trauma patients as well as perioperative management are concerned, almost $50 \%$ of trainees answered that their confidence was lower compared with the time period before the disease started.
Fig. 3 Number of operations assisted based on years of training in 4-month periods before and after the start of COVID-19

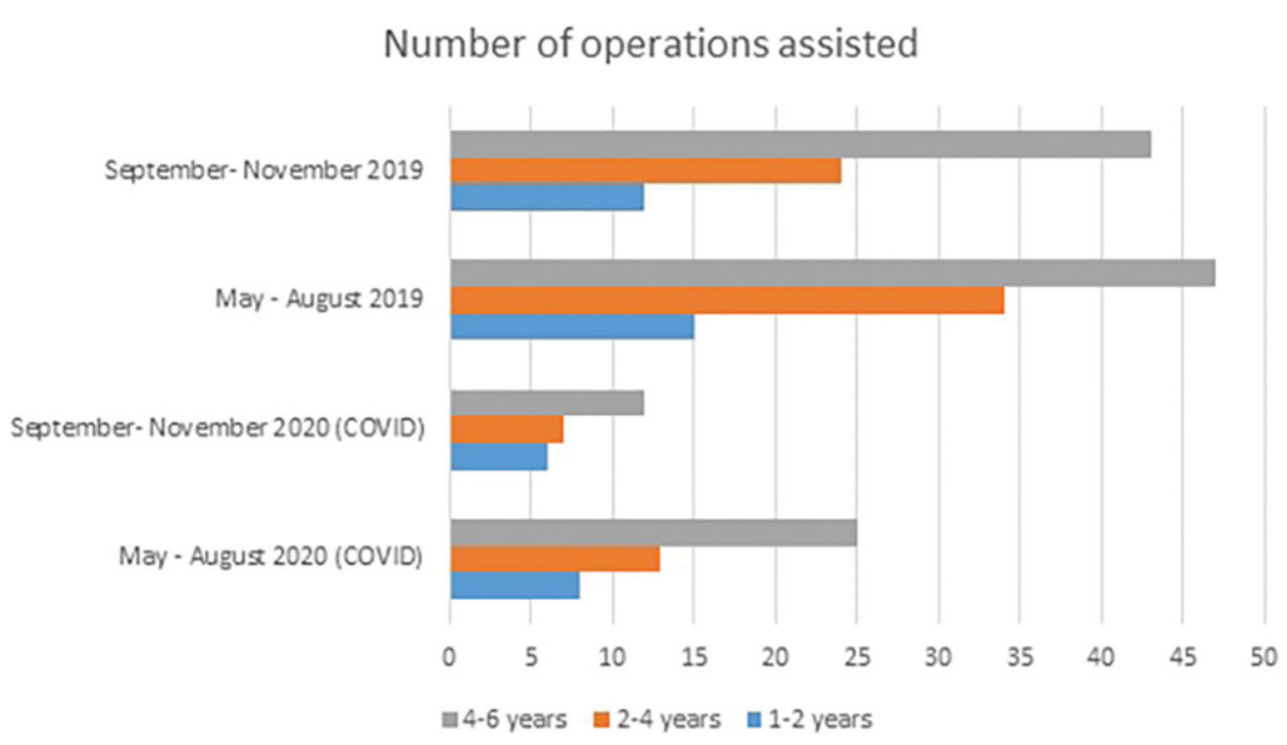


Fig. 4 Change in courses, conferences and exams attended before and after the start of COVID-19

\section{COURSES-CONFERENCES-EXAMS}

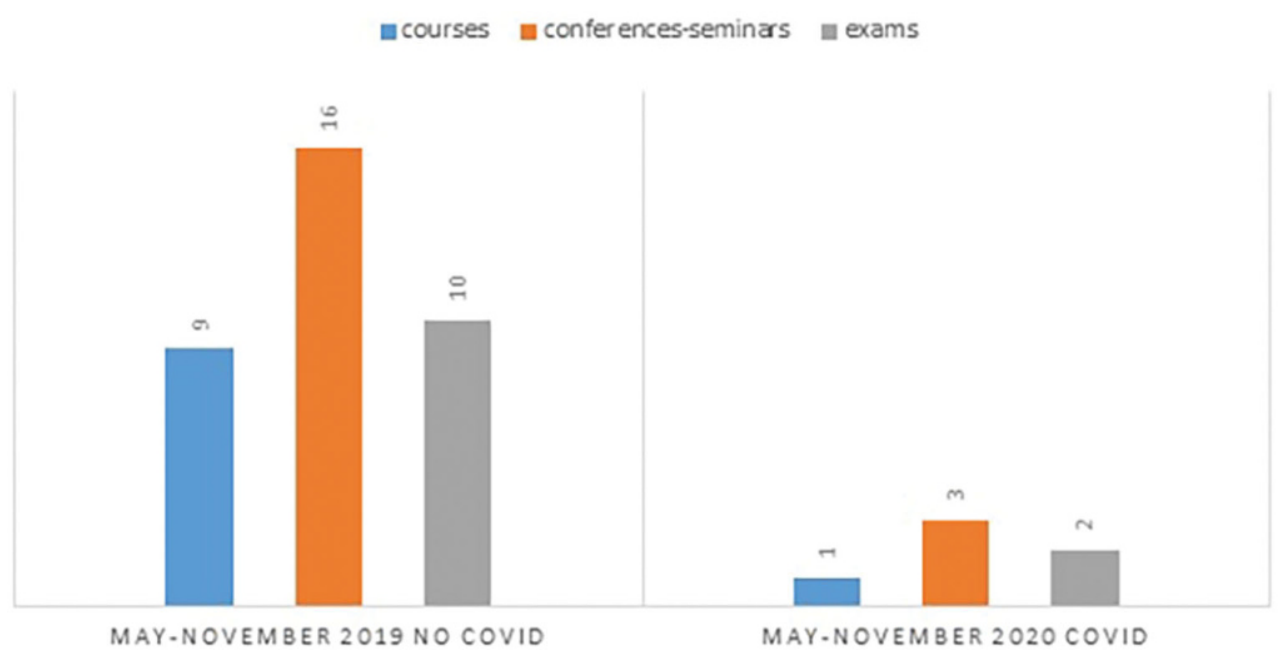

Fig. 5 Change in confidence in clinical practice before and after the start of COVID-19

\section{Confidence levels}

12

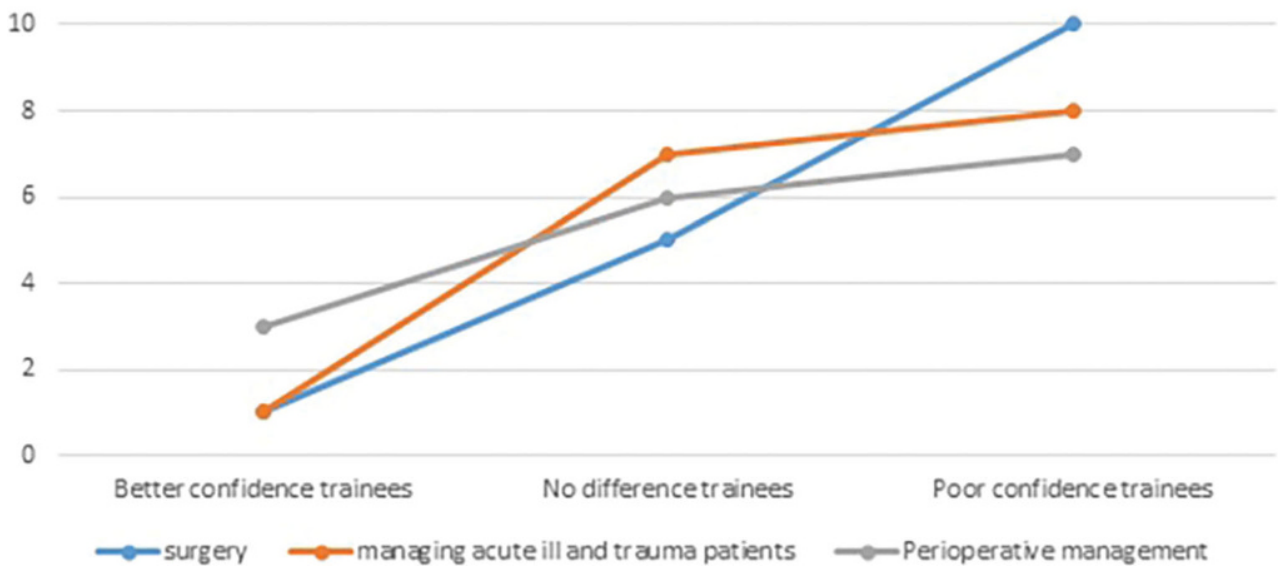

\section{Stress, anxiety levels and wellbeing}

Four (25\%) trainees felt significant stress levels as a result of the national lockdown. Interestingly enough, despite the fact that their workload had reduced, three $(18.7 \%)$ felt completely burned out. A total of $15(93.7 \%)$ answered that they had grave concerns about not being sufficiently competent as consultant surgeons due to the lack of adequate training during the pandemic. Fortunately, none of the trainees has been infected by the virus to date.

\section{Discussion}

The global COVID-19 pandemic has created unprecedented disruption to the surgical training of residents throughout the world. Various scientific committees are attempting to develop new strategies and programs to address this problem that puts the young generation of doctors at risk $[10,11]$. Non-emergency operative experience has been severely impacted and, to a lesser extent, experience in emergency cases. The present study highlights the impact of the pandemic on a tertiary hospital in Greece that covers a population of about $1,200,000$ people. The survey results are indicative of not only the impact on the training and education of young surgical trainees, but also of the impact on their confidence and stress levels. This agrees with the findings of other investigators in published studies from several countries [12-14].

The American College of Surgeons and the Royal College of Surgeons of England both recommend that non-emergency procedures be delayed, except operations in cancer patients [15]. Limiting the performance of elective clinical cases protects patients from in-hospital viral transmission and makes wards and critical care beds available for possible upsurges in the numbers of SARS-CoV-2 cases. As such, surgical trainees do not have the opportunity for exposure to cases and operations in which they could be in- 
volved in a non-pandemic era. This study demonstrates that young surgeons assist or perform almost $50 \%$ fewer operations when compared to their practice before COVID. Also apparent and alarming is the fact that not only their operative skills, but also their academic skills are lacking due to the restriction measures. Conferences and seminars are reduced in numbers and only available online. Moreover, stress levels that are closely associated with the mental health of young doctors are seriously affected-a situation that might pose a serious threat for social interaction in our communities.

It is imperative for surgical educators to design and implement new alternative ways to assist surgical trainees in their education. The use of technology could be of great importance to this end, with online platforms a major tool for their continuous learning. Homemade laparoscopic simulators could represent an important tool for the ongoing improvement of practical skills [9]. Trainers must provide trainees with academic opportunities that continue despite COVID-19, such as virtual conferences, which could be important in addressing the perception that access to academia has decreased $[12,16,17]$. Needless to say, educators should show flexibility in terms of the young surgeons' completion of their portfolios. Psychological support must also be provided for junior health care professionals experiencing stress during this period [18].

In a country like Greece, in a status post-major economic crisis with a depleted workforce and an increasing number of young scientists emigrating to other countries at a time when more workforce is required, this situation may become deleterious for the national health care system. It is true to say that the problem of young surgeons' inadequate education is a global issue and it is the duty of surgical educators to change the way training has traditionally been delivered and to help shape the future of medical education $[19,20]$.

This study, although limited by its descriptive nature, describes and suggests possible solutions to the health care problem that has evolved during the pandemic. Although the number of participants is limited, the results agree with existing evidence in the medical literature. There was sufficient time for respondents to have observed the changes that were apparent in this study. Moreover, given the fact that restriction measures were still in place at the time of administering the questionnaire, recall bias is significantly reduced.

Although it is not possible to assess the long-term results of the COVID-19 pandemic on the surgical training and education of young doctors, the present study, which is in agreement with other published studies, highlights the alarming nature of this problem in surgical practice. It is essential to maintain the integrity of surgical training, thereby protecting trainees while at the same time ensuring the sustainability of health system workforces.
As surgical specialties are largely dependent on hands-on experience, it is of great importance to implement new ways of learning practical skills. In addition, despite the fact that many academic programmes are now conducted virtually, there are other aspects of training, such as ward rounds, for which it would be relatively difficult to find a suitable substitute. In the same way that our existence during this pandemic relies on medical science, every health care system relies on their young doctors.

Author Contribution MV, MS, FM, ND, EA made substantial contributions to the conception, design and writing of the work, while IM supervised this research project.

\section{Declarations}

Conflict of interest M. Vailas, M. Sotiropoulou, F. Mulita, N. Drakos, E. Ambalov and I. Maroulis declare that they have no competing interests.

Ethical standards For this article no studies with human participants or animals were performed by any of the authors. All studies mentioned were in accordance with the ethical standards indicated in each case.

\section{References}

1. Zhu N, Zhang D, Wang W, Li X, Yang B, Song J, et al. A novel Coronavirus from patients with pneumonia in China, 2019. NEngl J Med. 2020;382(8):727-33.

2. Mohapatra RK, Pintilie L, Kandi V, Sarangi AK, Das D, Sahu $\mathrm{R}$, et al. The recent challenges of highly contagious COVID19, causing respiratory infections: Symptoms, diagnosis, transmission, possible vaccines, animal models, and immunotherapy. Chem BiolDrug Des. 2020;96(5):1187-1208.

3. Nassar AH, Zern NK, McIntyre LK, Lynge D, Smith CA, Petersen RP, et al. Emergency restructuring of a general surgery residency program during the Coronavirus disease 2019 pandemic: the University of Washington experience. JAMASurg. 2020;155(7):624-7.

4. Horesh D, Brown AD. Traumatic stress in the age of COVID19: A call to close critical gaps and adapt to new realities. Psychol Trauma. 2020;12(4):331-5.

5. Amparore D, Claps F, Cacciamani GE, Esperto F, Fiori C, Liguori G, et al. Impact of the COVID-19 pandemic on urology residency training in Italy. Minerva Urol Nefrol. 2020;72(4):505-9.

6. Al-Jabir A, Kerwan A, Nicola M, Alsafi Z, Khan M, Sohrabi C, et al. Impact of the Coronavirus (COVID-19) pandemic on surgical practice-Part 1. Int J Surg. 2020;79:168-79.

7. Hau HM, WeitzJ, BorkU. Impact of the COVID-19 Pandemic on Student and Resident Teaching and Training in Surgical Oncology. J Clin Med. 2020;9(11):3431.

8. Kogan M, Klein SE, Hannon CP, Nolte MT. Orthopaedic Education During the COVID-19 Pandemic. J Am Acad Orthop Surg. 2020;28(11):e456-e64.

9. Chick RC, Clifton GT, Peace KM, Propper BW, Hale DF, AlseidiAA, etal. Using technology to maintain theeducation of residents during the COVID-19 pandemic. J Surg Educ. 2020;77(4):729-32.

10. Ellison EC, Spanknebel K, Stain SC, Shabahang MM, Matthews JB, Debas HT, et al. Impact of the COVID19 pandemic on surgical training and learner well-being: 
report of a survey of general surgery and other surgical specialty educators. JAm Coll Surg. 2020;231(6):613-26.

11. Ferrel MN, Ryan JJ. The impact of COVID-19 on medical education. Cureus. 2020;12(3):e7492.

12. Khan KS, Keay R, McLellan M, Mahmud S. Impact of the COVID-19 pandemic on core surgical training. Scott Med J. 2020;65(4):133-7.

13. Zingaretti N, Contessi Negrini F, Tel A, Tresoldi MM, Bresadola V, Parodi PC. The impact of COVID-19 on plastic surgery residency training. Aesth Plast Surg. 2020;44(4):1381-5.

14. Shafi AMA, Atieh AE, Harky A, Sheikh AM, Awad WI. Impact ofCOVID-19 on cardiac surgical training: Our experience in the United Kingdom. J Card Surg. 2020;35(8):1954-1957.

15. Adesunkanmi AO, Ubom AE, Olasehinde O, Wuraola FO, Ijarotimi OA, Okon NE, Ikimalo JI, Fasubaa OB, Adesunkanmi ARK. Impact of the COVID-19 Pandemic on Surgical Residency Training: Perspective from a LowMiddle Income Country. World J Surg. 2021;45(1):10-17.

16. Fitzgerald JE, Giddings CE, Khera G, Marron CD. Improving the future of surgical training and education: consensus recommendations from the Association of Surgeons in Training. Int J Surg. 2012;10(8):389-92.
17. Juprasert JM, Gray KD, Moore MD, Obeid L, Peters AW, Fehling D, et al. Restructuring of a general surgery residency program in an epicenter of the Coronavirus disease 2019 pandemic: lessons from new York city. JAMA Surg. 2020;155(9):870-5.

18. The L. COVID-19: protecting health-care workers. Lancet. 2020;395(10228):922.

19. Ehrlich H, McKenney M, Elkbuli A. We Asked the Experts: Virtual Learning in Surgical Education During the COVID19 Pandemic-Shaping the Future of Surgical Education and Training. World J Surg. 2020;44(7):2053-2055.

20. Pawlik TM, Tyler DS, Sumer B, Meric-Bernstam F, Okereke IC, Beane JD, et al. COVID-19 pandemic and surgical oncology: preserving the academic mission. Ann Surg Oncol. 2020;27(8):2591-9.

Publisher's Note Springer Nature remains neutral with regard to jurisdictional claims in published maps and institutional affiliations. 